\title{
Diffusion tensor imaging and its application in navigation assisted surgery
}

\author{
Keqin Shen', Han Wang ${ }^{2}$, Yuxuan Wang ${ }^{3}$, Jincai Chang ${ }^{4}$, Jianzhong Cui ${ }^{5}$ \\ ${ }_{1,2,3,4}$ College of Sciences, North China University of Science and Technology, Tangshan, 063210, China \\ ${ }^{4} 3 \mathrm{D}$ Modeling and Application Innovation Laboratory, North China University of Science and Technology, \\ Tangshan, 063210, China \\ ${ }^{5}$ Department of Neurosurgery, Tangshan Gongren Hospital, Tangshan, 063000, China \\ ${ }^{4,5}$ Corresponding author \\ E-mail: ${ }^{1}$ shen990520@163.com, ${ }^{2973864902 @ q q . c o m, ~}{ }^{3}$ wyxuan666@163.com, ${ }^{4}$ jincai@ncst.edu.cn, \\ 5zhcui0001@163.com
}

Received 23 December 2019; received in revised form 1 June 2020; accepted 12 June 2020 DOI https://doi.org/10.21595/chs.2020.21262

Check for updates

Copyright (C) 2020 Keqin Shen, et al. This is an open access article distributed under the Creative Commons Attribution License, which permits unrestricted use, distribution, and reproduction in any medium, provided the original work is properly cited.

\begin{abstract}
Navigation-assisted surgery is being studied and used by more and more doctors because of its high success rate, and many techniques are needed, among which diffusion tensor imaging (DTI) plays an important role. The navigation assisted surgery system integrated with DTI will be more perfect and powerful. In order to prove this, we first successfully made DTI images of patients in 3D Slicer. After careful observation and study, we found that in navigation assisted surgery, doctors can use DTI to observe the location of brain tissue more clearly. This paper mainly studies the principle and data formula of DTI, and compares and summarizes how the nerve bundle image generated by DTI plays a role in navigation assisted surgery. The method proposed is very workable for doctors, and the effect of operation is also very easy to see.
\end{abstract}

Keywords: diffusion tensor imaging, navigation assisted surgical system, FA, diffusion, the tumor.

\section{Introduction}

Basser et al. [1] first introduced DTI into the field of MRI since 1994. DTI technology has developed rapidly and its application scope in medical field has been more and more extensive over the past 20 years. DTI technology is a new way to describe the structure of the brain and is a special form of magnetic resonance imaging (MRI). If MRI tracks hydrogen atoms in water molecules, for example, diffusion tensor imaging maps the direction water molecules move. Diffusion tensor imaging (which is presented in a different way than previous images) can reveal how brain tumors affect nerve cell connections and lead to brain surgery, guide medical personnel to perform brain surgery. This paper will discuss the role of DTI in brain tumor navigation surgery, and its preoperative modeling and navigation process are based on software 3D Slicer.

\section{The concept of diffusion tensor imaging}

\subsection{Diffusion}

Diffusion is the random and irregular movement that points to molecule, it is the physiology activity of human body important, it is one of means of material transport inside body, say Brownian motion again. Dispersion is a physical process whose primary force is the heat energy of molecules. In solution, factors that affect the dispersion of molecules include weight, intermolecular interaction (i.e. viscosity), and temperature.

Dispersion is a three-dimensional process, molecular one direction along the space dispersion distance is equal or unequal, diffuse way can be divided into two kinds: one kind is to point to in full uniform medium, movement of molecules with no obstacles, the distance of movement in all directions are equal, the dispersion mode called isotropic (isotropic) dispersion, such as water 
molecules in pure water dispersion for isotropic diffusion, namely in the brain tissue, cerebrospinal fluid and brain gray matter water molecules diffuse approximate isotropic diffusion. The other kind of dispersion is directional dependent, as in tissues arranged in a certain direction, where the molecules scatter at unequal distances in all directions, as anisotropic dispersion.

\subsection{Diffusion tensor imaging}

Diffusion Weighted Imaging is the development and deepening of Diffusion Weighted Imaging (DWI). It is the only non-invasive examination method that can effectively observe and track the white matter fiber bundles. By 2015, it will be mainly used to observe and track the white matter tract in the brain, study brain development and cognitive function, pathological changes of brain diseases, and preoperative planning and postoperative evaluation of brain surgery.

Diffusion imaging in magnetic resonance imaging, the contrast of tissues is not only related to the T1 and T2 relaxation times and proton density of tissues within each pixel, but also to the dispersion of water molecules within each pixel of the examined tissues. Hahn first proposed the effect of dispersion of water molecules on magnetic resonance signals in 1956.

\subsection{Dispersion coefficient}

The dispersion process can be measured by the diffusion-sensitive gradient magnetic field. When the gradient magnetic field is applied, the random movement of water molecules can obtain the random displacement, leading to the phase loss and the attenuation of the spin echo signal. The value that measures the size of the dispersion is called the dispersion coefficient, and is expressed as $D$, which is the average range of free random dispersion movement per unit time of a water molecule. The larger the $D$ value, the stronger the diffusion motion of water molecules. It can be described by a formula $\ln \left(S / S_{0}\right)=-b D$.

$D$ in the human body physiological environment value is influenced by many factors, so the common apparent diffusion coefficient (ADC) to measure the dispersion of water molecules in human tissue environment movement, movement of all the factors that affect water molecules (random and nonrandom) are stacked into a observations, displacement Strength of Water Molecule Reflecting the Direction of Diffusion Sensitive Gradient. According to Stejiskal-Tanner formula, $A D C=\frac{\ln \left(S_{2} / S_{1}\right)}{b_{1}-b_{2}}, S_{2}$ and $S_{1}$ are the signal strength under different $b$ values. Magnetic resonance DWI USES ADC value distribution imaging. The higher the ADC value, the stronger the diffusion of water molecules in tissues, and the lower the ADC value, the higher the signal on DWI.

The apparent dispersion coefficient ADC only represents the dispersion characteristics of water molecules in the direction of the application of a diffuse gradient magnetic field. The anisotropy of different tissues cannot be evaluated completely and correctly. Higano et al. applied a diffused gradient magnetic field on the $X, Y$ and $Z$ axes respectively in the study of determining the characteristics of diffuse anisotropy of the internal capsule and the radiation crown in patients with stroke and brain tumor. But the study results show that the organization of the three directions calculated diffusion weighted imaging anisotropic degree often underestimated, measured values are often rotate variable (that is, the value with the dispersion and the direction of the magnetic field be checked within the patient's position and direction), because most of the white matter fiber channel often tilt in the magnetic field direction coordinates, so that from one or three directions on diffusion gradient magnetic field right evaluation is not symmetrical anisotropic characteristics of the organizational structure. 


\section{Principles of diffusion tensor imaging}

\subsection{Dispersion anisotropy}

Dispersion is a kind of random, collision and transcendental motion of matter molecules in nature, namely Brownian motion. The dispersion of free molecules in the pure liquid is isotropic. The average distance of dispersion is only related to the properties of liquid molecules and the average temperature. The average free path of a free molecule in the liquid is measured by the dispersion coefficient (in $\mathrm{mm}^{2} / \mathrm{s}$ ). Water molecules in brain tissue are also continually diffusing, but it is not only affected by the characteristics of the tissue cells themselves, but also by the internal structure of the cells, such as the sheath, cell membrane, white matter fiber bundles. In a fixed sequence of organization structure, such as nerve fiber bundles, dispersion of water molecules in each direction is different, the water molecules are usually more inclined to go along nerve fiber bundle [2] to diffuse the direction, and rarely along the direction perpendicular to the nerve fiber bundle of dispersion, with the directional dependence of dispersion is called diffusion anisotropy.

\subsection{Diffusion weighted magnetic resonance imaging}

DWI is a relatively new technique to measure the micro-random displacement motion of spin protons. By 2015, it is mainly used to measure the movement of water molecules in vivo. The contrast of images is mainly related to the displacement motion of water molecules. The characteristics of movement of water molecules can be used on the direction of diffusion sensitive gradient apparent diffusion coefficient [3] of (Apparent Diffusion Coefficient, ADC) said. ADC value is a scalar, which only represents the dispersion characteristics of water molecules in the direction of the dispersion gradient magnetic field, but cannot completely and correctly evaluate the anisotropy characteristics of different tissues.

\subsection{Diffusion tensor imaging}

DTI is an advanced form of diffusion imaging that quantitatively evaluates the anisotropy of white matter (Fig. 1). In this imaging method, not only a single gradient pulse, but at least 6 non-collinear directions are required to apply diffusivity sensitive gradients. The simplest solution is $X, Y, Z, X Y, X Z$ and $Y Z$ directions. The second-order dispersion tensor [4] is a $3 \times 3$ matrix, and the off-diagonal items in the matrix can be eliminated by a mathematical method called similarity transformation. This redirects the $z$-axis of the voxel to the main direction of the white matter tract. This direction is called the major eigenvector. The dispersion coefficient in this direction is called the principal eigenvalue, and in addition to the principal eigenvector and eigenvalue, the new eigenvector is described in directions perpendicular to the new $Z$-axis (the new $X$ and $Y$ axes).

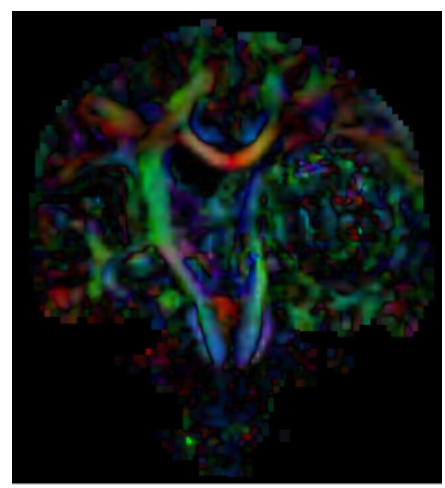

Fig. 1. DTI images 


\section{Relevant data parameters of diffusion tensor technique}

\subsection{Average dispersion rate}

Mean diffusivity (MD) is used to evaluate the diffusion of a voxel or region in a tissue, it is necessary to eliminate the influence of anisotropic dispersion and express it with a constant parameter, that is, the change of this parameter does not depend on the direction of dispersion. The trace of the diffusion tensor is an invariant parameter among the elements of the diffusion tensor, $\operatorname{Tr}(D)=D_{X X}+D_{Y Y}+D_{Z Z}$, mean diffusivity $M D=\frac{1}{3} \operatorname{Tr}(D)=\frac{1}{3}\left(D_{X X}+D_{Y Y}+D_{Z Z}\right)$. MD reflects the overall dispersion level of the molecule (the size of the mean ellipsoid) and the overall dispersion resistance. MD only indicates the size of the dispersion, not the direction of the dispersion. The larger the MD, the more free water molecules there are in the tissue.

\subsection{Degree of anisotropy}

The degree of anisotropy reflects the degree of displacement of molecules in space and is related to the direction of tissues. Fractional anisotropy (FA), relative anisotropy (RA), and volume ratio index (VR) are used to quantitatively analyze anisotropy. These exponentials are calculated from the eigenvalues $\left(\lambda_{1}, \lambda_{2}\right.$ and $\left.\lambda_{3}\right)$ of the dispersion tensor.

(1) FA: Partial anisotropy index, which is the ratio of anisotropic components of water molecules to the whole dispersion tensor, varies from 0 to 1.0 means that dispersion is unrestricted. For example, FA value of CSF is close to 0 . For very regular directional tissues, the FA value is greater than 0 , for example, the FA value of brain white matter fibers is close to 1 .

The calculation formula of FA value is as follows:

$F A=\frac{\sqrt{3}}{2} \cdot \frac{\sqrt{\left(\lambda_{1}-D\right)^{2}+\left(\lambda_{2}-D\right)^{2}+\left(\lambda_{3}-D\right)^{2}}}{\sqrt{\lambda_{1}^{2}+\lambda_{2}^{2}+\lambda_{3}^{2}}}$.

(2) RA: relative anisotropic index, which is the ratio of the anisotropic part of the dispersion tensor to the isotropic part of the dispersion tensor, varies from 0 (isotropic dispersion) to $\sqrt{2}$ (infinite anisotropy).

The calculation formula of RA is as follows:

$R A=\frac{\sqrt{\left(\lambda_{1}-D\right)^{2}+\left(\lambda_{2}-D\right)^{2}+\left(\lambda_{3}-D\right)^{2}}}{\sqrt{3 D}}$.

(3) VR: volume ratio index. Is the ratio of the volume of the ellipsoid to the volume of the sphere. Since it varies from 1 (i.e. isotropic dispersion) to $0,1 / V R$ is more likely to be applied clinically.

The calculation formula of VR is as follows:

$V R=1-\frac{\lambda_{1} \lambda_{2} \lambda_{3}}{D^{3}}$

\subsection{The main direction of dispersion}

The main direction of diffusivities, also known as the principal axis of the ellipse of the diffusion tensor, reflects the spatial orientation of the organizational structure.

Although there are many parameters reflecting anisotropy, FA value has been applied more clinically by 2015 , for the following reasons:

(1) The FA image can provide a better contrast between grey matter and grey matter, and it is 
easy to select the area of interest, so that the FA value measured is more accurate.

(2) FA value does not change with the rotation direction of the coordinate system, and FA value is the physical characteristics of the organization. Values obtained from the same object at different times, different imaging equipment and different objects are comparable.

\section{The role of diffusion tensor imaging in neuronavigation-assisted surgery}

We quoted a patient and analyzed the role of DTI in preoperative preparation and operation.

\subsection{The role of DTI in preoperative}

Before traditional craniocerebral surgery, doctors use two-dimensional CT images to make operation plans according to their own experience. Before the minimally invasive puncture and removal of intracranial hematoma [5], doctors used the module to perform the general registration (elastix) in 3D slicer. CT images and DTI images (Fig. 2), DTI images and MRI images (Fig. 3) [6] can be fused to clearly see the white matter and functional areas of the patient's brain. 3D slicer software can see the sagittal position, coronal position and axial position of the fusion image, also can use the $3 \mathrm{D}$ reconstruction of the fusion image, and make it a complete $3 \mathrm{D}$ reconstruction system.

The longest axis of hematoma can be selected by naked eyes, and the approximate puncture point and direction can be determined by the longest axis [7]. According to the three-dimensional images of other structures in the brain, the final puncture path and puncture point should be determined to avoid puncture deviation. It leads to the situation that the operation cannot be carried out, so the operation plan can be formulated.

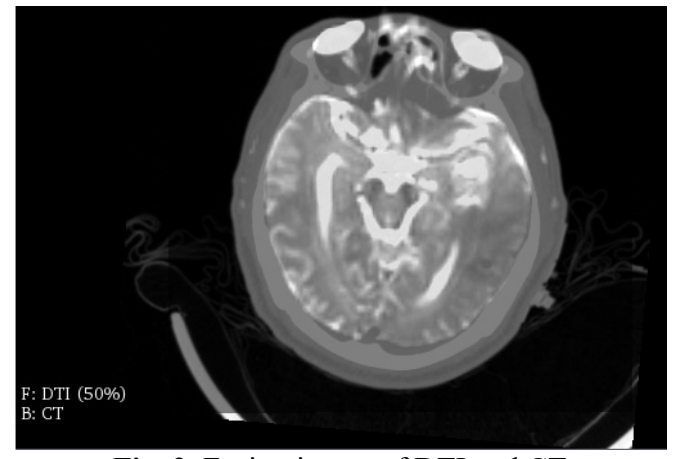

Fig. 2. Fusion image of DTI and CT

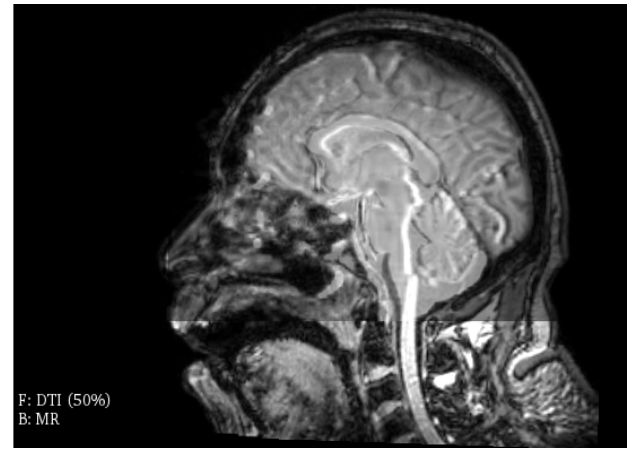

Fig. 3. Fusion of DTI and MR

\subsection{The role of DTI in intraoperative navigation}

The traditional method of minimally invasive puncture for the removal of intracranial hematoma is CT localization [8]. The puncture section of hematoma in CT scan was directly "copied" to the patient's scalp. According to the location of the metal marker, the puncture point can be accurately determined, and its positioning effect is more precise and accurate. But this method needs repeated scanning, which increases the exposure times and dose. To some extent, it depends on the experience of doctors. Another important point is that there are many nerve bundles around the hematoma of the patient. These nerve bundles play an important role in language, movement, etc. they cannot be damaged. This method alone can't guarantee to avoid damaging nerve bundle completely. DTI was used to make the nerve and corticospinal tract model [9] of the patients. In order to improve the success rate of the operation, the navigation system is introduced to display the intracranial condition in real time. 


\subsubsection{Nerve fiber bundles}

Fiber bundle refers to the fiber bundle (conduction bundle) in the central nervous system, where the nerve fibers with the same starting, ending, course and function gather and walk together. In the 3D Slicer software, the patient's fiber bundle model is generated through DTI image through the "Tractography Seeding" module, and it is displayed at the same time. As shown in Fig. 4 and Fig. 5, the fiber bundle around the tumor can be observed obviously. Therefore, the doctor can avoid the fiber bundle and reduce the patient's injury by fine-tuning the direction of the needle during the operation, thus making the operation more successful.

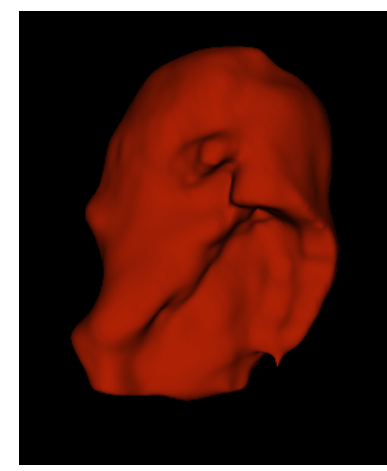

Fig. 4. The patient's hematoma model

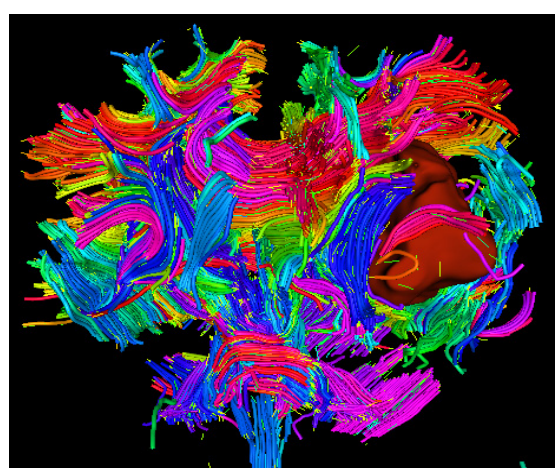

Fig. 5. Patient's hematoma and nerve tract

\subsubsection{Corticospinal tract}

Among the numerous fibers, corticospinal tract system is a kind of nerve tract that doctors need to pay special attention to. Its neurons are located in the motor area of the anterior central gyrus and other areas of the cortex. Its fibers gather in the posterior foot of the ipsilateral internal capsule and descend to the medulla oblongata to form a cone.

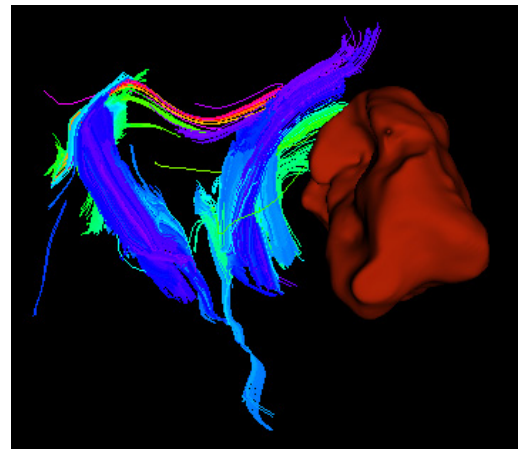

Fig. 6. Patient's hematoma and corticospinal tract mode

Most of the fibers cross to the opposite side at the lower end of the cone, forming the corticospinal lateral tract, descending at the lateral cord of the spinal cord, and directly or indirectly ending at the motor neurons of the anterior horn. A small part of the uncrossed fibers in the pyramids form the corticospinal tract, which also directly or indirectly terminates in the anterior horn cells. The function of this bundle is to control the voluntary movement of skeletal muscle, which together with the anterior horn motor neurons constitute the conduction pathway of voluntary movement. Therefore, if it is damaged, it will cause limb motor dysfunction and affect the quality of life of patients. 3D Slicer can display the patient's corticospinal tract through the module "Tractography Seeding". During the operation, the image files of corticospinal tract 
are imported into the navigation assisted operation platform, and the doctors can observe while doing, so as to help the doctors better complete the operation.

\section{Conclusions}

DTI is the only noninvasive method to trace the white matter fiber bundle. In the preoperative planning, DTI can determine the location of the nerve bundle. In operation navigation, DTI can effectively avoid damaging nerve bundle. After operation, DTI can reduce the treatment of postoperative nerve injury. It has a profound impact on the study of brain function and gradually extends to other systems of the body. With the continuous development and improvement of science and technology, the technical level of DTI will continue to develop and improve, I believe that the surgical navigation technology will be more perfect.

\section{Acknowledgements}

This work was supported by the National Science Foundation of China (51674121, 61702184), Tangshan Innovation Team Project (18130209B, 18130210B), the Hebei Key Laboratory of Science and Application, Research and Practice Project of Higher Education Teaching Reform in Hebei Province (2017GJJG111) and Ministry of Education Production University Cooperation Education Project (201802305012).

\section{References}

[1] X-ray Diagnostics Writing Group. X-ray Diagnostics (Volume 3). Shanghai Science and Technology Publishing House, Shanghai, 1986, p. 1202.

[2] Wang J. L., Xie J. X. Anisotropy of Water Diffusion in Adult Brain Tissues. Chinese Journal of Radiology, Vol. 33, Issue 10, 1999, p. 675.

[3] Wang X. H., Huang Li, Liu S. R., Chen H. F. Study on diffusion weighted images of human brain in different diffusion gradient directions. Chinese Medical Imaging Technology, Vol. 1, 2002, p. 82-84.

[4] Zhang W. D., Liang B. L., Chen J. Y., et al. Determination and analysis of diffusion tensor imaging parameters of brain structure in normal adults. Journal of Clinical Radiology, 2004.

[5] Wei J. W., Chen S. L. Minimally invasive removal of intracranial hematoma in the treatment of 60 cases of hypertensive intracerebral hemorrhage. Chinese Journal of medicine and clinical practice, Vol. 16, Issue 8, 2016, p. 1176-1177.

[6] Liu J. H., Xie G. X., Liu Hao, Chen D. Y., Tang X. N., Lei Zheng, Yu S. Y., Wu H. B. Current application of diffusion tensor magnetic resonance imaging technology. Guangxi Medical Science, Vol. 40, Issue 17, 2018, p. 2004-2006+2009.

[7] Zhou Z. H., Liu X. D. Application of image fusion in surgical navigation. Computer Engineering and Application, Vol. 31, 2004, p. 31-32.

[8] Wen Kui CT stereotactic guided minimally invasive puncture for the removal of intracranial hematoma and craniotomy for the treatment of hypertensive cerebral hemorrhage. Clinical Practice, Vol. 10, 2012, p. 7-10.

[9] Xiao Hui, Chen Z. Q., Zhang B. Y., et al. The significance of three-dimensional tractography in the evaluation of corticospinal tract injury in patients with acute cerebral infarction. Chinese Journal of Clinical Rehabilitation, Vol. 34, 2006, p. 78-80+202.

[10] Hu W. C. Application of MR diffusion tensor imaging combined with nerve fiber tracing technology in the diagnosis and treatment of cerebral infarction. Chinese Medical Equipment, Vol. 30, Issue 8, 2015, p. 43-45. 


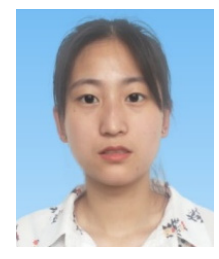

Keqin Shen is a junior majoring in information and computing science at North China University of technology. She is interested in 3D modeling. In this paper Keqin Shen responsible for methodology, formal analysis, data curation, writing-original draft preparation.

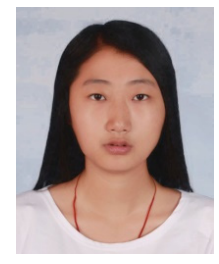

Han Wang is a student of North China University of Science and Technology, majoring in information and computing science and working in 3D modeling laboratory. She has a strong interest in 3D medical imaging. In this paper Han Wang responsible for methodology, formal analysis, writing-review and editing.

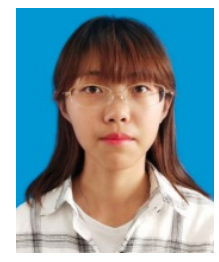

Yuxuan Wang is a student of North China University of Science and Technology, majoring in information and computing science and working in 3D Modeling Laboratory. She is interested in 3D modeling. In this paper Yuxuan Wang responsible for methodology, formal analysis, visualization.

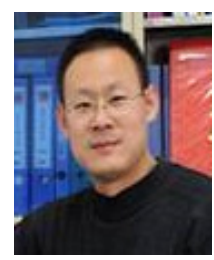

Jincai Chang, Ph.D., is now the dean of the Department of Information and Computing Science at North China University of Technology. In this paper Jincai Chang responsible for supervision, funding acquisition.

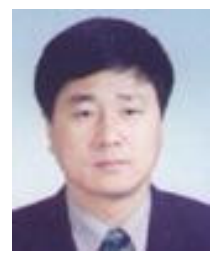

Jianzhong Cui is now working in Tangshan Workers Hospital, chief physician and Professor. He is good at surgical treatment of craniocerebral trauma and cerebrovascular diseases. In this paper Jianzhong Cui responsible for conceptualization, resources. 